\title{
Mobile Hospitalization for Kidney Transplantation
}

\author{
Iraky Khalifa \\ Department of Computer \\ Science, Faculty of Computers \\ and Information, Helwan \\ University, Egypt
}

\author{
Hala Abd Al-Glil \\ Department of Computer \\ Science, Faculty of Computers \\ and Information, Helwan \\ University, Egypt
}

\author{
Mohamed M. Abbassy \\ Department of Information \\ Technology, Faculty of \\ Computers and Information, \\ Helwan University, Egypt
}

\begin{abstract}
Due to the rapid development of hospital system, doctors have an urgent need to access Electronic Medical Record system (EMR) through smart phone and tablets, in wireless environments. Since most of the EMR systems are based on Windows Operating System. The mechanisms of making EMR mobiles become an important research issue in smart hospital. In this study an intelligent decision support system based on fuzzy logic was designed and used for the kidney transplantation operations, through smart phone and tablets, in wireless environments. This proposed system is an integrated system utilizes rule based expert system and fuzzy logic and used for monitoring and early notification for kidney transplant patients and their donors by using smart phones and tablets for any operating system.
\end{abstract}

\section{Keywords}

Intelligent decision support system, Fuzzy logic, Mobile Hospital, Smart Mobile Hospital System (SMHS), Electronic Medical Record (EMR), Mobile kidney transplantation

\section{INTRODUCTION}

Mobile computing and mobile devices enhanced the relationship between humans and computers. They introduced a new form of communication based on context. A new form of communication allows people to interact easily with the object, computer, environment and others. Development of mobile devices and corresponding medical software applications creates opportunities for hospitals to better organize, store, and share medical data using mobile technology [1].

Moreover, medical information systems (MISs) may include medical imaging storage and transmission systems, nursing information systems, laboratory information systems, and pharmacy information systems. To treat patients, medical personnel can use different information systems in accordance with their needs, in order to diagnose and run tests, like blood tests, urine sampling, computed tomography scans, X-ray [2].

A medical information system produces all kinds of medical information in various formats, including texts, numbers, pictures, and static and dynamic images. This heterogeneous information can then be integrated without the need of medical personnel. According to a patient ID, name, or other basic data, the information can be indexed by, for medical use upon request [3].

In addition, artificial intelligence (AI) has significantly contributed in the evolution of medical informatics and biomedicine since it provides a variety of tools available to be exploited, from rule-based expert systems and fuzzy logic to neural networks and genetic algorithms. The earliest work in medical AI dates back to the early 1970s, when the field of AI was about 15 years old. Since then, there is a growing interest in the application of AI techniques in biomedical engineering and informatics, ranging from knowledge-based reasoning for disease classification to learning and discovering novel biomedical knowledge for disease treatment, indicative of the maturity and influence that have been achieved to date [4]. The integration of AI techniques in biomedical engineering and informatics is presented, especially in the following core topics: (a) feature selection, (b) visualization, (c) classification, (d) data warehousing and data mining, and (e) analysis of biological networks [5].

Therefore, this study aims to building Smart Mobile Hospital System "SMHS", based on web applications, Smartphones, and an interconnection with the Cloud Computing, used for monitoring, early notifying and optimizing the process of managing kidney transplantation. This design system will be applied in kidney transplantation for monitoring and analyzing bioanalysis for kidney transplant patients and their donors. In addition, this system will be used to analyzing biomedical to help doctor to take decision.

\section{RELATED WORK AND BACKGROUND}

There are several academic and industrial features in this field. The healthcare sector is not only widely distributed and fragmented, but it also exhibits a high degree of heterogeneity with strong local autonomy [6]. The current lack of standards across different institutions prevents from using a single software solution to support a co-operative working environment [7]. It is also important to realize that the domain is very information-intensive and mission-critical [8]. The large volume of medical data with its complexity and variety makes it impractical to be transported between 'point-ofsource' and 'point-of-delivery' as in the traditional clientserver case.

Monitoring plays an important role during patient stay in hospital for continuous medical assistance and treatment. For this purpose, cabled measurement equipment is already used to insure reliable control of vital signs. However such a process complicates patient autonomy or mobility. To overcome this problem, wireless technologies can be applied and permits mobile healthcare monitoring [9].

Moreover, decisions play an important role in medicine, especially in medical diagnostic processes. Decision Making Support System (DMSS) helping physicians are becoming a very important part in medical decision making, particularly in those situations where decision must be made effectively and reliably $[10,11]$.

The significance of medical decision making can be divided into three facts: (I) demand of the medical field itself; (II) impact of social cost; (III) maintenance of public health and 
medical care safety. For different clinical requirements, there have been different designs and applications. In short, the main objective of the medical decision support system is to use computer technology to help physicians and other medical personnel improve medical service quality with lower cost [12].

Fuzzy logic is not logic that is fuzzy, but logic that is used to describe fuzziness. Fuzzy logic is the theory of fuzzy sets, sets that calibrate vagueness and based on the idea that all things admit of degrees. Fuzzy logic is a set of mathematical principles for knowledge representation based on degrees of membership. Unlike two-valued Boolean (i.e., conventional) logic, fuzzy logic is multi-valued. It deals with degrees of membership and degrees of truth. Fuzzy logic uses the continuum of logical values between 0 (completely false) and 1 (completely true) which means it may accept things with partly true and partly false at the same time [13].

\section{PROPOSED SYSTEM}

Proposed system is called Smart Mobile Hospital System "SMHS" is gather Artificial Intelligent (AI), providing access to end users (doctor and nurse) via smart phone and tablets by their authority, monitor kidney patient and their donors by notification between end users to communicate, and this system stored cloudly to gain feature of cloud. "SMHS" used for monitoring and analyzing Bioanalysis and measuring biomarkers of kidney transplant patients and their donors by using smart phones and tablets for any operating system. Besides, this system also includes the patients' injection entry records, medicines and prescriptions.

\subsection{System Architecture}

The SMHS was used to determine early warnings from the kidney transplant patients and kidney donated patients'. The design system utilizes the Service Oriented Architecture (SOA). This system consists of web applications, original mobile applications, used by smart phones and tablets for any operating system. Those applications are closely connected to web services and decision support systems finally received by the end users, whom are the doctors and the nurses. The system provides those following services: Electronic medical records (EMRs) of the kidney transplant patients and kidney donated patients are achieved and managed by doctor who is responsible for each patient, recorded each important signs performed for both kidney patients and their donors : biomarkers and Bioanalysis, such as blood pressure, blood glucose, height, and weight, Heart Rate, SPO2, Temperature, Systolic blood pressure, Creatinine, Fasting plasma glucose and Bun), Finding out of range measurement values, and fire notification via DSS, Building kidney donated patients' and kidney transplant patients' profiles depending on DSS, mostly used in kidney transplantation, notification module, to connection between the end users.

The "SMHS" is applied through client-server architecture. Authenticated end users have the total privilege to easily and smoothly access the integrated system via client applications; web applications, smart phones and tablets based on role based security. Role based security for authorized access to the system is implemented to handle the security and privacy. There are different roles defined like Doctor and Nurse. Depending on the type of query like SELECT, UPDATE, $\mathrm{ADD}$ and the role defined to a user, he will be granted/denied access to the EMR of a particular patient.

Figure 1 shows the overall picture of the system architecture of proposed "SMHS" End user must have authentication access to interact with the "SMHS" in order to retrieve the data from web service. The web service sends/receives the data of the patient to end users "Doctor, nurse ", the end users ask the required data from web service then send this request to database server provides the required data and send it back to webserver. Then the webserver send it to the web service in which it will be appear on end users screen through "SMHS".

If the end user is a doctor who has the authenticate to take decision concerning kidney transplantation ,the web service is going to receive all the required data, from doctor

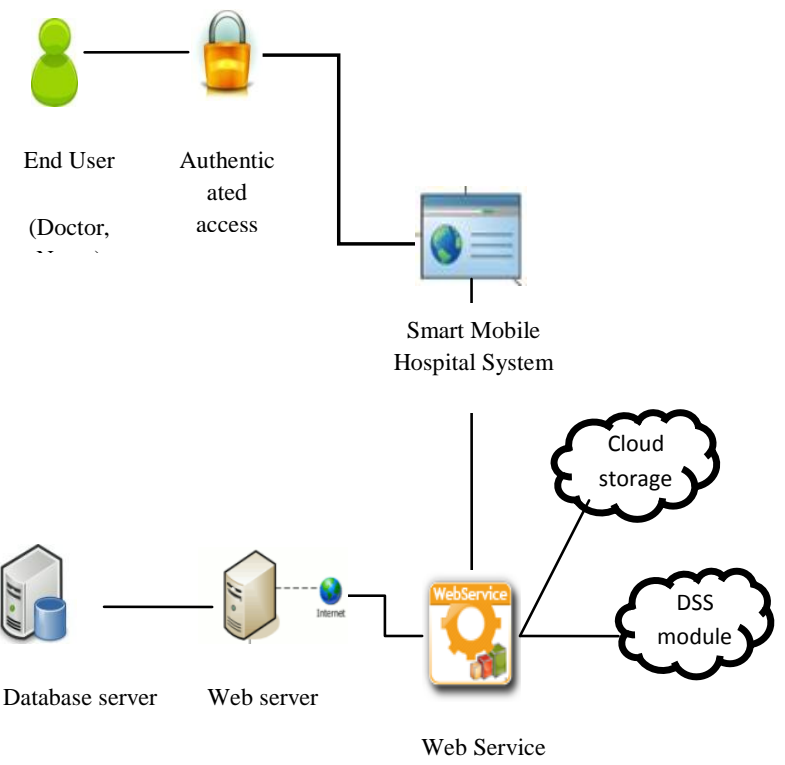

Fig1: The Architecture of "SMHS".

concerning the kidney transplant patient and the kidney transplant donor, and concerning also their analysis and Bioanalysis. The web service will send all those data to Decision Support System "DSS" module. The DSS will support the doctor decision regarding kidney transplantation. Besides, these data will be automatically stored cloudly through the cloud storage via web service.

\subsection{System Functionality}

This section describes the analysis of the "SMHS" and the services it offers to the end users (Figure 2). The description of the system's functionality and offered services will be achieved via the existence of the following services: 


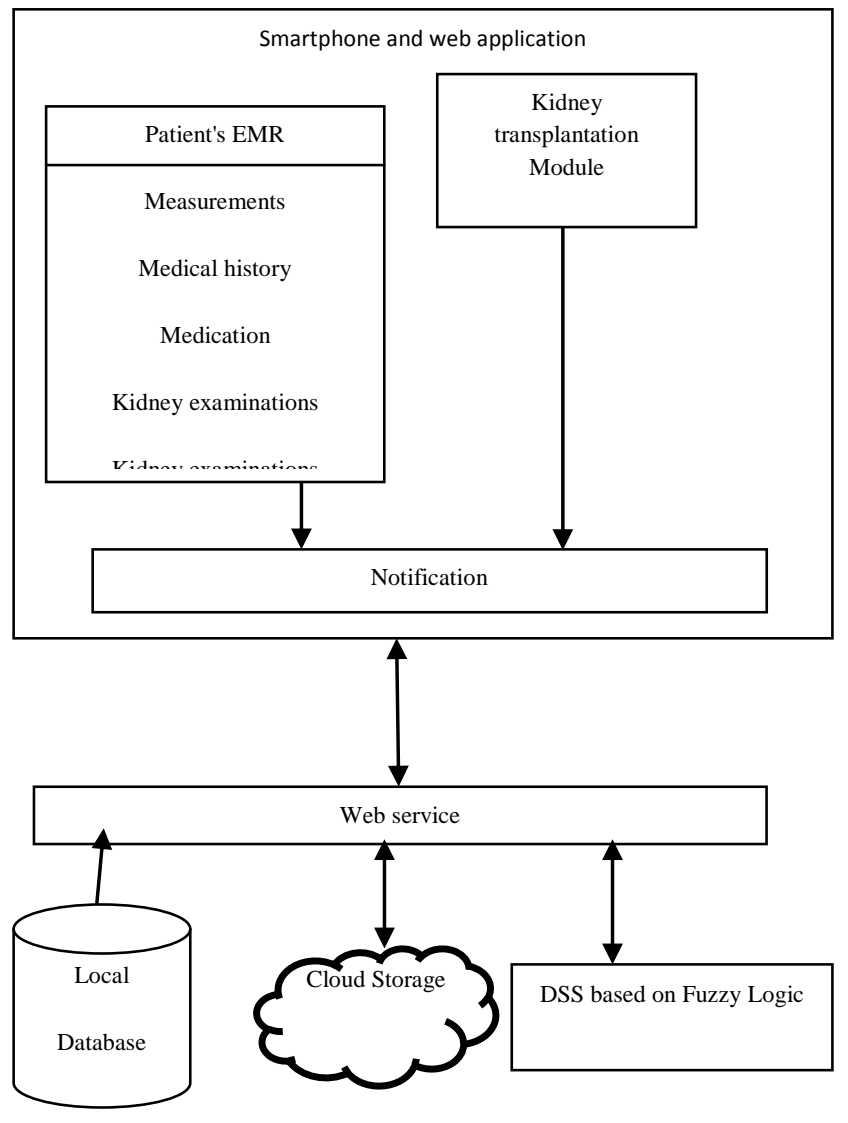

Fig2. Smart Mobile Hospital System "SMHS” Services

\section{a) Electronic Medical Record (EMR):}

Doctors can access their kidney transplant patients' and their kidney donated patients' medical records by using the "SMHS". The EMR of this system consists of the patient's medical history, medication, Bioanalysis, kidney examinations (physical checkup), and periodical measuring Biomarkers.

First, Medical History for kidney donor for kidney diseases It consists of detailed information about hypertension, kidney failure, increased risk for chronic kidney disease, kidney damage with normal or elevated Glomerular filtration rate (GFR) , kidney damage with mildly decreased (GFR) , Moderately decreased (GFR), severely decreased (GFR), and kidney disorders.it includes some basic information about cholesterol, diabetes mellitus, tobacco use, thyroid disease, cancer lung/liver/kidney neurological/ /gastrointestinal/autoimmune/hematoloc.

Second, kidney transplant patients' medical history. It is created in details from the first time the patient visits the hospital. The medications could be updated by the doctors. In addition to the patients' information, it could be updated by the nurses. Bioanalysis information examinations are recorded and stored into "SMHS" by doctors or Bioanalysis staff.

Third, Medication It includes all the previous and current medicines, dosage, route, duration and instructions of the kidney transplant patients and the kidney donated patients. Furthermore, doctors would be able to follow up their kidney transplant patients' treatments and medical services. Moreover, they would be able to send back or not. Bioanalysis include information for complete blood count, coagulation times, creatinine, Fasting plasma glucose, and Systolic blood pressure, and Blood urea nitrogen (Bun), and biochemical
Fourth, Measuring Biomarkers and Bioanalysis are performed by Nurse and medical technician. The measurement types and their frequency are made according to the kidney transplant patients' and kidney donated patient's health conditions. There are some types like blood pressure, blood glucose, height, and weight, Heart Rate "HR", SPO2, Temperature "TEMP", Systolic blood pressure, Creatinine, Fasting plasma glucose and Bun and the frequency of measurements.

Most of the previous informations are stored cloud, including medication, Bioanalysis, periodical measuring, and demographics. This information will be also available for the third parties, so that they can take advantage features of cloud computing. For instance, improving efficiency in the use of medical resources, cost, and will be easy to expand, depending on health information and user needs.

b) Kidney transfer Modules:

Depending on the experts' knowledge and experiences, kidney transplant cases are divided into several modules. The kidney transplant modules are, in fact, enhanced kidney transplant patient and donor profiles that comprise decision support systems (DSSs), based on fuzzy logic. Fuzzy logic is used because, it offers solutions when a system is so complicated that cannot be mathematically modeled, or when it presents fuzziness. In this System, we take that the blood pressure, blood glucose, height, and weight, Heart Rate "HR", SPO2, Temperature "TEMP", Systolic blood pressure, Creatinine, Fasting plasma glucose and Bun and other Bioanalysis as the most major parameters. These parameters are determined from having several interviews with professional experts. Thus, the previous parameters will be used as the input to the system design. The fuzzy set is shown in TABLE 1 .

Table 1. Range of Fuzzy Values

\begin{tabular}{|c|c|c|}
\hline $\begin{array}{c}\text { Fuzzy } \\
\text { Variables }\end{array}$ & $\begin{array}{c}\text { Linguistic } \\
\text { values }\end{array}$ & Fuzzy range \\
\hline \multirow{2}{*}{ Creatinine } & Normal & $<1.4 \mathrm{mg} / \mathrm{dl}$ \\
\cline { 2 - 3 } & Abnormal & $>1.4 \mathrm{mg} / \mathrm{dl}$ \\
\hline \multirow{2}{*}{$\begin{array}{c}\text { Fasting } \\
\text { plasma } \\
\text { glucose }\end{array}$} & Normal & $<103 \mathrm{mg} / \mathrm{dl}$ \\
\cline { 2 - 3 } $\begin{array}{c}\text { Systolic } \\
\text { blood } \\
\text { pressure }\end{array}$ & Abnormal & $>103 \mathrm{mg} / \mathrm{dl}$ \\
\cline { 2 - 3 } & Normal & $<123 \mathrm{mmHg}$ \\
\hline \multirow{2}{*}{ Bun } & Normal & $>123 \mathrm{mmHg}$ \\
\cline { 2 - 3 } & Abnormal & $<9 \mathrm{mg} / \mathrm{dl}$ \\
\hline \multirow{2}{*}{ Sodium } & Normal & $136-146 \mathrm{mEq} / \mathrm{L}$ \\
\cline { 2 - 3 } & Abnormal & $\begin{array}{c}>146 \mathrm{mEq} / \mathrm{L} \\
\text { And }<136 \mathrm{mEq} / \mathrm{L}\end{array}$ \\
\hline \multirow{2}{*}{ Potassium } & Normal & $3.5-5.0 \mathrm{mEq} / \mathrm{L}$ \\
\cline { 2 - 3 } & Abnormal & $<3.5 \mathrm{mEq} / \mathrm{L} \mathrm{and}$ \\
& Normal & $4-6 \mathrm{mEq} / \mathrm{L}$ \\
\cline { 2 - 3 } & Abnormal & $\begin{array}{c}<4 \mathrm{mg} / \mathrm{dl} \\
\text { and }>6 \mathrm{mg} / \mathrm{dl}\end{array}$ \\
\hline \multirow{2}{*}{ Uric Acid } & Normal & $3.5-5.0 \mathrm{gm}$ \\
\hline \multirow{2}{*}{ Albumin } & Abnormal & $>5.0 \mathrm{mg}$ and $<3.5 \mathrm{mg}$ \\
\hline
\end{tabular}


c) Notification Module:

It is a module of "SMHS" that optimizes the communication between the end users. Real time notification will alert $\mathrm{him} / \mathrm{her}$ before the patient treatment by 10 minutes then five minutes. Those notifications used to remind the nurse by patients' treatment's time and doses. The nurse has to check after performing any action. Furthermore, the doctor could checkup and monitors his nurse's work through those real time notifications. These will be sent to doctor automatically by the system. Through those notifications, doctors will be able to know whether the patient took the treatment on time or not. All those facilities and services are to be applied in an efficient way to saving time and resources wasted currently in such cases.

\subsection{System Development}

This system was applied based on PHP and Mysql language. Mysql and PHP are together regarded as an easy and powerful way to create dynamic web pages that really interact with the medical staff. HTML can create well formatted web pages. Besides PHP and Mysql, we can gather data for medical staff, create special content on the fly, and do many other difficult things that can't be handled alone by HTML. The design of PHP is considered a unique merit, as it is designed to a company HTML. The main aim in using this platform is to solve the application building problem in iPad, iPhones, Android, Windows Mobiles, and so on.

The integrated development environment (IDE) facilitated user interface design, code editing, testing and debugging in a consolidated window. The IDE was installed on a laptop Table 2.

Table 2. Development and Testing Environment

\begin{tabular}{|c|c|c|}
\hline Feature & Smart Phone & Tablet \\
\hline Model & $\begin{array}{c}\text { IPhone, Samsung } \\
\text { Galaxy S III, } \\
\text { Nokia Lumina }\end{array}$ & IPad , Samsung tablet \\
\hline OS & \multicolumn{2}{|c|}{ iOS , Android and windows } \\
\hline
\end{tabular}

\section{SYSTEM IMPLEMENTATION}

This section describes the implementation of the design System "SMHS". The limitations of the screen size of smart phones and tablets, how to arrange presented contents in a screen is an issue. The screen was seen as too small requiring too much time to enter data quickly. The use of thumb keyboards was an option but nurses demonstrated a preference for not entering text using these formats. So users and their tasks should be paid more attention to in the arrangement of an interface and the switch mode between menu and toolbar is introduced. Also, graphics, colors and appearances would affect applications; the interface is designed to be concise and identical to Windows series which are familiar to users. Moreover, combos and check boxes are adopted for reducing the operation of manual typing.

Thus, the doctors can easily find the patients' name list, data of medical treatments. Moreover, doctors could check whether the patient took the medicine on time or not. Same to nurses, they can also easily find their patients' name list. Through this system, they can receive doctors' orders and execute the order by using the medical order checking module. By just one click on the patients' name the nurses can receive the doctors' orders.
This system starts by a login page as shown in figure 3 . Inside this login, there are the users name, password and user's type; whether $\mathrm{s} / \mathrm{he}$ is a doctor or nurse. If a doctor log into his/her page, it will appear only his/her patients' name besides the notifications. Moreover, his patient's recent changes, development and treatment will be shown by real time notifications. Because this system is mainly applied by kidney transfer cases, thus; the patient's and the volunteer's tests will be highlighted by different colors and will be indicated by (below Normal, Normal, above Normal) . On the other hand, if a nurse log into his/her page, it will appear only his/her patients' name, their treatment's time and doses. Moreover, real time notification will alert her before the patient treatment by 10 minutes then five minutes. Those notifications used to remind the nurse by patients' treatment's time and doses. The nurse has to check after performing any action.

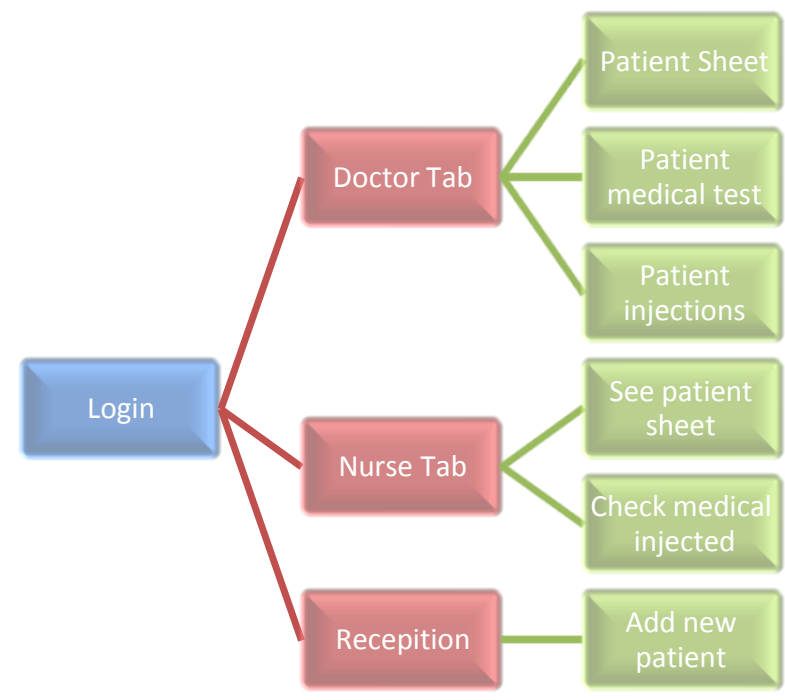

Fig 3: System Design

In this study, cloud technology was used to build "SMHS" because the clouds can vastly benefits in hospital work. There are some of the advantages of this system, such as storing information in the "SMHS" based on cloud gives unlimited storage capacity. Hence, no more need to worry about running out of storage space or increasing your current storage space availability. Since all data are stored in the cloud, backing it up and restoring the same is relatively much easier than storing the same on a physical device. Therefore, this makes the entire process of backup and recovery much simpler than other traditional methods of data storage. In addition, this technology helps to access the information from anywhere, where there is an Internet connection.

The "SMHS" provides us groups of identical benefits, those benefits are; it helps in the clinical practice that occur every day via the end users "doctors, nurses". Moreover, the kidney transplant module forms, and DSS are used as guidance to follow the kidney transplant patient and his donor in the kidney transplant operations, standardizing in this way the tasks that have to be done for every patient, each day and by whom. By using notification module help to get fast briefing reduces mistakes and speeds up the process management. 


\section{CONCLUSION}

Smart Mobile Hospital System "SMHS" is an important aspect in the Mobile Health Care field by providing the ability to review by a smart phone or a tablet. This proposed approach presents, to the doctor, new medical patterns. Doctors will be able to access their electronic medical records at the patients' bedside via smart phones and tablets. This system is regarded as an integrated system, based on web applications, Smartphones, and tablets an interconnection with the Cloud Computing to take advantages such as storing information, easy backup and recovery and can access the information from anywhere, and this system used for monitoring Kidney transplant patients, donor and early notifying and optimizing the process of managing Kidney transplantation. The system supports kidney transplantation modules based on Bioanalysis and customized DSS based on fuzzy logic. In contrast with the old traditional system, the Smart Mobile Hospital System "SMHS" reduces the burden on doctors, saves time and effort for nurses, improves the efficiency of medical treatment, and finally, enhances a great accurate and safe care upon the patient.

\section{REFERENCES}

[1] Figueiredo, C., Nakamura, E., Computação Móvel. 2003 Novas Oportunidades e Novos Desafios T\&C Amazônia.

[2] E. Faliagka, V. N. Syrimpeis, A. Tsakalidis, G. K. Matsopoulos, J. Tsaknakis, and G. Tzimas, "Diagnosis: A global alignment and fusion medical system," in Proceedings of the 3rd International Conference on Health Informatics, HEALTHINF 2010, pp. 21-28, esp, January 2010.

[3] C. H. Liu, Y. F. Chung, T. W. Chiang, T. S. Chen, and S. D.Wang, "Mobile agent approach for secure integrated medical information systems," Journal of Medical Systems, vol. 36, no.5, pp. 2731-2741, 2012.

[4] V. L. Patel, E. H. Shortliffe, M. Stefanelli et al., "The coming of age of artificial intelligence in medicine," Artificial Intelligence in Medicine, vol. 46, no. 1, pp. 517, 2009.
[5] Y. Peng, Y. Zhang, and L. Wang, "Artificial intelligence in biomedical engineering and informatics: an introduction and review," Artificial Intelligence in Medicine, vol. 48, no. 2-3, pp. 71-73, 2010.

[6] J.Grimson; G.Stephens; B.Jung; W.Grimson; D.Berry And S.Pardon, 2001."Sharing Health-care Records over the Internet", IEEE Internet Computing, Vol. 5, No. 3 pp. $49-58$.

[7] .Bilykh, Y.Bychkov, D.Dahlem, JH.Jahnke, G.McCallum, C.Obry, A.Onabajo, C. Kuziemsky, 2003. "Can GRID Services Provide Answers to the Challenges of National Health Information Sharing?", proceedings of the Centre for Advanced Studies conference on Collaborative Research, Toronto, pp.39-53.

[8] J.Grimson, W.Grimson, W.Hasselbring, 2000. "The SI Challenge in Health Care", Communications of the ACM, pp. 48-55.

[9] Paksuniemi, M.; Sorvoja, H.; Alasaarela, E.; Myllyla, R.; "Wireless sensor and data transmission needs and technologies for patient monitoring in the operating room and intensive care unit," Engineering in Medicine and Biology Society, 2005. IEEE-EMBS 2005. 27th Annual International Conference of the, pp.5182-5185, 17-18 Jan. 2006.

[10] V.Podgorelec, P.Kokol,B.Stiglic, I.Rozman,"Decision trees: an overview and thier use in medicine",Journal of Medical system, vol26,pp445-463,oct.2002

[11] C.Marsala, "A Fuzzy Decision Tree Based Approach to Characterize Medical Data , " in Proc.2009 IEEE Int.Conf. on Fuzzy System, Jeju island,Korea, pp13321337

[12] C. C. Chang, "A computer-aided medical decision support tool for treatment risk analysis," The Journal of China Association for Medical Informatics, No.7, 1998 pp.14-25.

[13] M. Negnevitsky, Artificial Intelligence- A Guide to Intelligent Systems, 2nd Ed, Addison Wesley, 2005. 